\title{
MAGNETOSPHERIC MULTISCALE (MMS) MISSION ATTITUDE GROUND SYSTEM DESIGN
}

\author{
Joseph E. Sedlak, ${ }^{(1)}$ Emil Superfin, ${ }^{(2)}$ and Juan C. Raymond ${ }^{(2)}$ \\ (1) a.i. solutions, Inc., 10001 Derekwood Lane, Lanham, MD 20706, USA, joseph.sedlak@ai-solutions.com \\ (2) a.i. solutions, Inc., 10001 Derekwood Lane, Lanham, MD 20706, USA, emil.superfin@ai-solutions.com \\ ${ }^{(3)}$ NASA/Goddard Space Flight Center, Code 591, Greenbelt, MD 20771, USA, juan.c.raymond@nasa.gov
}

Keywords: MMS, attitude, ground support

\begin{abstract}
This paper describes the attitude ground system (AGS) design to be used for support of the Magnetospheric MultiScale (MMS) mission. The AGS exists as one component of the mission operations control center. It has responsibility for validating the onboard attitude and accelerometer bias estimates, calibrating the attitude sensors and the spacecraft inertia tensor, and generating a definitive attitude history for use by the science teams.

NASA's Goddard Space Flight Center (GSFC) in Greenbelt, Maryland is responsible for developing the MMS spacecraft, for the overall management of the MMS mission, and for mission operations. MMS is scheduled for launch in 2014 for a planned two-year mission.

The MMS mission consists of four identical spacecraft flying in a tetrahedral formation in an eccentric Earth orbit. The relatively tight formation, ranging from 10 to $400 \mathrm{~km}$, will provide coordinated observations giving insight into small-scale magnetic field reconnection processes. By varying the size of the tetrahedron and the orbital semi-major axis and eccentricity, and making use of the changing solar phase, this geometry allows for the study of both bow shock and magnetotail plasma physics, including acceleration, reconnection, and turbulence. The mission divides into two phases for science; these phases will have orbit dimensions of $1.2 \times 12$ Earth radii in the first phase and $1.2 \times 25$ Earth radii in the second in order to study the dayside magnetopause and the nightside magnetotail, respectively. The orbital periods are roughly one day and three days for the two mission phases.
\end{abstract}

Each of the four MMS spacecraft will be spin stabilized at 3 revolutions per minute (rpm), with the spin axis oriented near the ecliptic north pole but tipped approximately $2.5 \mathrm{deg}$ towards the Sun line. The main body of each spacecraft will be an eight-sided platform with diameter of $3.4 \mathrm{~m}$ and height of $1.2 \mathrm{~m}$. Several booms are attached to this central core: two axial booms of $14.9 \mathrm{~m}$ length, two radial magnetometer booms of $5 \mathrm{~m}$ length, and four radial wire booms of $60 \mathrm{~m}$ length. Attitude and orbit control will use a set of axial and radial thrusters. A four-head star tracker and a slit-type digital Sun sensor (DSS) provide input for attitude determination. In addition, an accelerometer will be used for closed-loop orbit maneuver control.

The primary AGS product will be a daily definitive attitude history. Due to power limitations, the star tracker and accelerometer data will not be available at all times. However, tracker data from at least 10 percent of each orbit and continuous DSS data will be provided. An extended Kalman filter (EKF) will be used to estimate the three-axis attitude (i.e., spin axis orientation and spin phase) and rotation rate for all times when the tracker data is valid. For other times, the attitude is generated by assuming a constant angular momentum vector in the inertial frame. The DSS sun pulse will provide a timing signal to maintain an accurate spin phase. There will be times when the Sun is occulted and DSS data is not available. If this occurs at the start or end of a definitive attitude product, then the spin phase will be extrapolated using the mean rate determined by the EKF. 
To improve the accuracy of the closed-loop orbit maneuver control, the accelerometer bias must be estimated whenever a burn is planned. A simple Kalman filter for the bias has been designed to work in parallel with the attitude and rate EKF. This bias filter will be implemented both onboard and on the ground. The AGS will use data downlinked prior to each maneuver to validate the onboard attitude and bias estimates. Figure 1 shows an example of the estimated bias minus the truth model value, which varies due to random walk. The first $1800 \mathrm{sec}$ are excluded from the plot to allow for convergence.

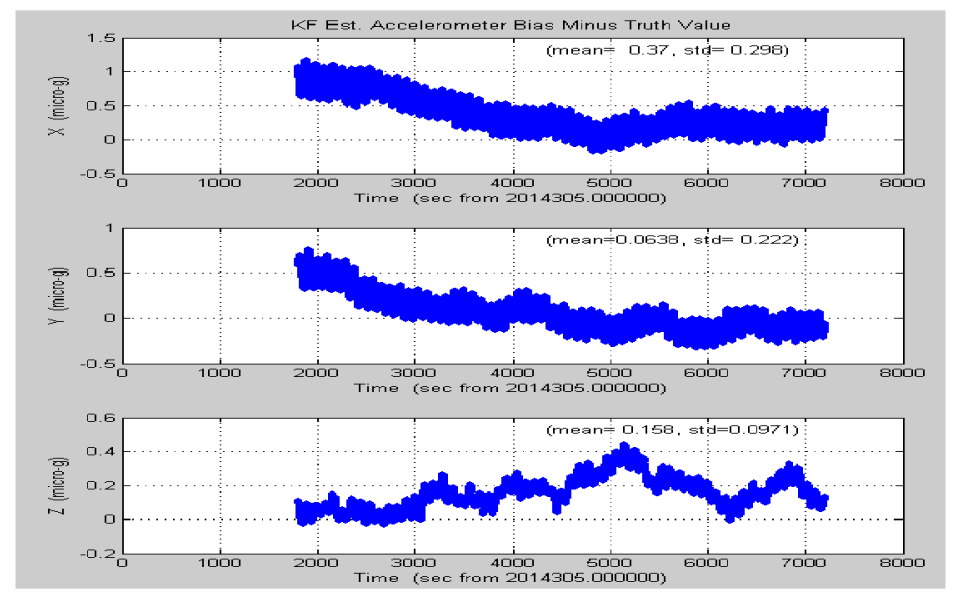

Fig. 1. Estimated minus truth model accelerometer bias (micro-g) vs. time.

The four separate star tracker fields of view must be calibrated on-orbit to correct for launch shift. The relative misalignment between any two heads can be computed by a least squares method (a very efficient algorithm for this is based on the well-known QUEST quaternion estimation algorithm). Once the relative misalignments are known, all four heads can be rotated to a common frame such that the effective body frame defined by the mean alignment is unchanged. This makes the least disruption to the definitive attitude history. Similarly, the DSS slit azimuth and elevation bias are computed relative to the tracker alignment; however, the DSS digitization may be too coarse to improve the elevation.

The current MMS spacecraft design does not include rate-sensing gyroscopes, so the EKF must use dynamical modeling for attitude and rate propagation. One consequence of this is that the accuracy of the transverse components of the estimated rotation rate, and the subsequent accelerometer bias estimates, are very sensitive to errors in the inertia tensor. No large attitude or spin rate maneuvers are planned after full boom deployment. Only two aspects of the inertia tensor are amenable to on-orbit estimation for a rigid-body spinning spacecraft without maneuvers. The first is the direction of the major principal axis in the body frame. Knowledge of this axis is necessary to account for coning error. The second is the ratio of the largest inertia eigenvalue to the mean of the smaller eigenvalues. For a rigid body, this ratio affects the nutation of the angular momentum vector in the body frame. The ratio can be determined by inspection of two particular frequencies in the attitude estimate. Both the coning and nutation corrections can be determined by iterating between the inertia calibration and the EKF estimates. However, MMS will not be a rigid body. In particular, there are important normal modes involving the 60 -meter wire booms having periods as long as $100 \mathrm{sec}$. Diaphragms included in the fuel tank design will limit fuel slosh (necessary for accurate orbit maneuver control), but will also limit system damping. Tests using high-fidelity simulation data show that the coning correction can be done even in the presence of large boom oscillations, but the nutation correction cannot.

The paper will describe the algorithm or system design for each of the AGS responsibilities. Test results will be presented for each functionality using data either from a simple rigid-body simulator or from a high-fidelity simulation that includes the multibody vibrations. 